\title{
FUNCIONAMIENTO FAMILIAR Y RENDIMIENTO ACADÉMICO EN LA FACULTAD DE CIENCIAS HUMANAS Y EDUCACIÓN DE UNA UNIVERSIDAD PRIVADA
}

\author{
Dámaris S. Quinteros Zúñiga*
}

\begin{abstract}
Resumen
La investigación tuvo como objetivo estudiar la relación entre las variables funcionamiento familiar y rendimiento académico de los alumnos del primer y segundo año de la Facultad de Ciencias Humanas y Educación de una universidad privada. El modelo del funcionamiento familiar sobre el cual se trabajó es el de McMaster (MMFF) propuesto y descrito por Epstein, Baldwing y Bishop (1983), quienes sostienen que el funcionamiento familiar está constituido por 7 dimensiones: solución de problemas, funcionamiento de roles, comunicación, respuesta afectiva, compromiso afectivo, control conductual y funcionamiento general. La escala utilizada para la medición del funcionamiento familiar fue el Family Assessment Device (FAD), elaborado en base al MMFF. Se consideró como indicador del rendimiento académico al promedio obtenido de las calificaciones de los estudiantes durante el primer ciclo de estudios del año 2008. Se analizó la relación entre cada una de las dimensiones y el rendimiento académico de una muestra compuesta por 193 estudiantes, encontrándose que los alumnos con un rendimiento académico alto califican a su familia como más funcionales en casi todas las dimensiones excepto en la dimensión funcionamiento de roles. Se concluye que el nivel de funcionamiento familiar sí se relaciona significativamente con el rendimiento académico de los alumnos del primer y segundo año de la Facultad de Ciencias Humanas y Educación, excepto en la dimensión funcionamiento de roles donde no se encontró una correlación significativa.
\end{abstract}

Palabras clave: funcionamiento familiar, rendimiento académico, familia y escuela.

\begin{abstract}
Abstrac
The present research aims to study the relationship between family functioning and academic performance variables of first and second term students of the Faculty of Education and Human Science in a private university. The MacMaster Model of Family Functioning (MMFF) described by Epstein, Baldwing and Bishop (19983) was used in this study. The model identifies seven dimensions on family functioning: problem solving, role functioning, communication, affective responsiveness, affective involvement, behavioral control and general functioning. The Family Assessment Device (FAD) scale based on the McMaster Model of Family Functioning (MMFF) was used for assesing family functioning dimensions. The academic performance average's indicator was obtained from the student's grades during the first term of studies in 2008.

The relationship among every dimension and the academic performance on a sample of 193 students was analyzed, in which the families of the students with high academic performance are more functional in almost all dimensions, except the one related to the role functioning. Therefore, the family functioning level has a significant relationship with the academic performance in the first and second term students of the School of Education and Human Sciences, with the exception of the role functioning dimension in which a significant correlationship was not found.
\end{abstract}

Key words: family functioning, academic performance, family and school.

\footnotetext{
* Magister en Psicología Educativa. Candidata al Doctorado en Psicología por la Universidad Femenina del Sagrado Corazón. LimaPerú. Docente en la Universidad Peruana Unión. damaris@upeu.edu.pe
} 


\section{INTRODUCCIÓN}

Una de las tareas importantes en el desarrollo del ser humano es su formación profesional y para ello, la vida universitaria viene a ser el tercer peldaño educativo que el estudiante debe alcanzar, luego de pasar por la primaria y la secundaria.

Para responder acertadamente a este desafío, las instituciones educativas hacen sus mayores esfuerzos por lograr un alto rendimiento académico en sus alumnos, considerando sus capacidades cognitivas, conductuales y socio espirituales. Sin embargo, en la tarea educativa cotidiana, los docentes pueden encontrarse con alumnos cuyo rendimiento académico es deficiente o bajo, lo que puede complicar la tarea formativa que tienen las instituciones educativas.

El análisis de los factores que condicionan el desempeño académico ha sido motivo de numerosas investigaciones, ya que la naturaleza de este proceso es multicausal. Siguiendo a Ortiz (1999), si se quiere reflexionar sobre la identidad personal, ¿por qué se es de una forma y no de otra?, ¿por qué se actúa de cierta manera?, ¿por qué uno se orienta en determinado sentido?, parte de esta respuesta está en la historia familiar de cada uno. Además, encontramos que para Andrade, Miranda y Freixas (2000) es un hecho cada vez más aceptado el rol fundamental de los padres en el desarrollo de ciertas capacidades cognitivas de sus hijos y, especialmente, la estimulación en la realización de tareas y actividades estudiantiles.

En este trabajo se pretende valorar el resultado académico tomando en cuenta los factores que pueden actuar como mediadores o determinantes de éxito o fracaso académico.

Al revisar la bibliografía se encuentran diversos estudios con el objetivo de identificar los factores que alteran el rendimiento académico, y dentro de ellos resaltan las características de los grupos familiares a los que pertenecen los estudiantes, ya que los problemas afectivo-familiares y la falta de un soporte familiar que coopere con su formación académica podrían complicar el panorama. Dentro de la dinámica de las familias, llama la atención el funcionamiento familiar, entendido como el conjunto de procesos de interacción y convivencia que se dan en el hogar y que generan sentimientos de seguridad, identidad y bienestar.
De modo que de la amplia gama de variables que pueden incidir en el rendimiento académico nos hemos centrado en la familia y específicamente en su funcionamiento. Para conocer la naturaleza de este fenómeno se ha tomado como grupo de estudio a los alumnos que cursan el primer y segundo año en la facultad de Ciencias Humanas y Educación, en las escuelas académico profesionales de Educación, Psicología y Ciencias de la Comunicación.

El objetivo es describir las características de su funcionamiento familiar según el Modelo McMaster de Epstein, Baldwing y Bishop (1983), basado en una perspectiva sistémica de la familia, y analizar la naturaleza de su relación con el rendimiento académico de los estudiantes.

Se considera relevante conocer esta relación de variables en el grupo de alumnos de los primeros años de la universidad, ya que ingresar a la universidad implica cambios, adaptación. Los primeros ciclos académicos conforman un periodo de acomodación para el alumno que debe aprender a manejarse independientemente tanto en el ámbito académico como en su vida personal. Por ejemplo debe aprender a reorganizar su tiempo, adecuarse a un nuevo lugar de vivienda, establecer nuevos vínculos socioafectivos y asumir responsabilidades personales (Universidad Nacional de Río Cuarto, 2008). Es de interés conocer si en este contexto que se encuentra el estudiante de los primeros años, el soporte familiar favorece el afrontamiento de los retos mencionados.

En ese sentido, surge la siguiente interrogante: ¿existe relación entre el funcionamiento familiar y el rendimiento académico de los alumnos del primer y segundo año de la facultad de Ciencias Humanas y Educación de la Universidad Peruana Unión?

\section{Funcionamiento Familiar}

Hurtado (2000) señala que la familia es uno de los grupos primarios de la sociedad que brinda experiencias básicas para las relaciones interpersonales y que estos grupos poseen rasgos dinámicos y cambiantes. Es de entender que la familia y el rol que desempeña han ido evolucionando, por factores culturales, sociales, políticos y económicos. Por lo tanto, conocer las características familiares permite comprender mejor las cualidades que presentan sus miembros en todos los ambientes con los que se relacionan, como el educativo, por ejemplo. 
Sauceda y Maldonado (2003) nos indican que desde la perspectiva sistémica, la familia no debe ser entendidaúnicamente en función de las personalidades de sus integrantes, sino en cuanto a las relaciones interpersonales y los procesos de interacción que existen entre ellos. Es decir que la familia viene a ser una red de comunicaciones e interacción en la cual cada familiar desde el más joven hasta el más viejo influye sobre el sistema entero y es influido por él. El sistema familiar experimenta una diferenciación y realiza sus funciones a través de subsistemas organizados por generación, sexo, intereses, etc. Así aparecen subsistemas como el de cónyuges, el formado por los varones, el de las mujeres, el de los hermanos, etc. Desde esta perspectiva, cuando un miembro de la familia presenta un síntoma psicológico, éste es entendido como conducta de reacción al contexto familiar y para tratar el síntoma es necesario cambiar la situación en la que vive e involucrar a otros integrantes de la familia.

Epstein y otros (1983) explican que cada familia adopta un determinado estilo de vida que se caracteriza por conductas altamente frecuentes y de esta manera es posible tipificarlas como funcionales o disfuncionales. La funcionalidad o disfuncionalidad de las relaciones familiares, dependen del grado de satisfacción de las necesidades biológicas, psicológicas y sociales de sus integrantes. La familia difiere de otros grupos humanos en muchos aspectos como la duración e intensidad de los lazos afectivos que unen a sus integrantes y las funciones que estos desempeñan. Así, las características psicológicas de la familia son más que la suma de las personalidades individuales de sus miembros. Para entender su funcionamiento se requiere conocer también sus relaciones interpersonales, pues las acciones de cada uno de ellos producen reacciones y contra reacciones en los demás y en ellos mismos.

Baldwing (1998) dice que el funcionamiento familiar puede evaluarse por la comunicación entre los miembros, el desempeño de roles, la respuesta afectiva, el involucramiento afectivo y el control de la conducta y flexibilidad. Y De la Cuesta, Pérez y Louro (1996) consideran al funcionamiento familiar, como la dinámica relacional interactiva y sistémica que se da entre los miembros de una familia y se evalúa a través de la categoría de cohesión, armonía, rol, permeabilidad, afectividad, participación y adaptabilidad. Sin embargo, el funcionamiento familiar no es un asunto de todo o nada, por lo que muchas veces se hace necesario cuantificarlo desde el grado óptimo hasta el más disfuncional.

\section{Modelo de funcionamiento familiar McMaster (MMFF)}

El MMFF focaliza las dimensiones del funcionamiento que han sido observadas y que tienen mayor impacto sobre la salud emocional y física o sobre problemas de los miembros de la familia. Cada dimensión ha sido definida desde lo más inefectivo hasta lo más efectivo. La hipótesis central es que un funcionamiento inefectivo o disfuncional contribuye a una presentación clínica, mientras que un modo más bien efectivo sostiene un estado de salud física e intelectual óptimo.

Las dimensiones del funcionamiento familiar descritas en el MMFF son las siguientes:

1. Solución de problemas: se refiere a la habilidad de la familia para resolver problemas en un nivel que mantenga un funcionamiento familiar efectivo.

2. Comunicación: se define la comunicación como el intercambio de información dentro de la familia. Se divide en dos áreas: instrumental y afectiva. La comunicación puede ser clara o encubierta, el continuo entre ambos indica si el contenido del mensaje es claramente expresado o si es camuflado, embrollado o vago; y el continuo entre la comunicación directa e indirecta indica si los mensajes van hacia el objetivo adecuado o si se distrae en otros.

3. Funcionamiento de roles: Ios roles familiares son definidos como los patrones repetitivos de conducta por los que los miembros cumplen las funciones familiares a fin de mantener un orden efectivo y sano. Se identifican cinco funciones necesarias: (a) provisión de recursos, (b) crianza y sostén, (c) satisfacción sexual de los adultos, (d) desarrollo personal de sus miembros (físico, emocional, educacional, social), (e) mantenimiento y manejo del sistema familiar, que incluye la toma de decisiones, los límites y la pertenencia, el control de la conducta, funciones económicas, problemas de salud.

4. Respuesta afectiva: es la habilidad para responder a un estímulo dado con calidad y cantidad de 
sentimientos apropiados. Se toman en cuenta dos aspectos importantes, la capacidad de responder con todo el espectro de sentimientos, y la emoción experimentada a tiempo con el estímulo y el contexto adecuado.

5. Compromiso afectivo: es la medida en que la familia muestra interés y valora las actividades de cada uno y se interesa en los miembros individuales de la familia; el monto de interés que cada miembro demuestra, la manera en que lo hace y cómo se apoyan unos a otros.

6. Control conductual: se define como las pautas que una familia adopta para sostener las conductas en tres áreas: situaciones de peligro físico, situaciones que implican encuentro y expresión de necesidades y energías psicobiológicas y situaciones que incluyen conductas de socialización interpersonal.

\section{Rendimiento Académico}

García (1987) señala que la conceptualización del rendimiento académico depende de las características de cada institución educativa y de sus objetivos. Por ello que se consideran distintas fuentes que permitan ampliar nuestro conocimiento sobre la naturaleza del rendimiento académico.

El rendimiento académico es entendido por Pizarro (1985) como una medida de las capacidades respondientes o indicativas que manifiestan, en forma estimativa, lo que una persona ha aprendido como consecuencia de un proceso de instrucción o formación.
El mismo autor, desde una perspectiva del alumno, define el rendimiento académico como la capacidad respondiente de éste frente a estímulos educativos, susceptible de ser interpretado según objetivos o propósitos educativos pre establecidos.

La bibliografía revisada conceptualiza al rendimiento académico como un fenómeno multicausal, donde no sólo se toma en cuenta las capacidades intelectuales o cognitivas del estudiante, si no que se incluyen aspectos sociales, familiares e institucionales y además siguiendo la idea de Garbanzo (2007), algunos de ellos, según su nivel de influencia, serán considerados como factores causales o mediadores.

\section{MÉTODO}

Diseño no experimental y de corte transversal porque no se manipularon las variables, y porque los datos fueron tomados en un momento dado. El tipo de estudio fue descriptivo - correlacional, ya que se describió el comportamiento del rendimiento académico y el funcionamiento familiar y la relación entre ellos (Cabrero y Richart, 2008).

\section{Participantes}

Lamuestra quedó conformada por 193 estudiantes: 24 de la EAP Educación, 39 de la EAP de Ciencias de la Comunicación y 130 de la EAP de Psicología, distribuidos de la siguiente manera (ver Tabla 1):

Tabla 1

Muestra de alumnos del primer y segundo año de la Facultad de Ciencias Humanas y Educación por carreras, durante el 2008-I.

\begin{tabular}{lccr}
\hline E. A. P. & Primer año & Segundoaño & Total \\
\hline Educación & 16 & 8 & 24 \\
Psicología & 68 & 62 & 130 \\
Ciencias de la comunicación & 21 & 18 & 39 \\
Total & 105 & 88 & 193 \\
\hline
\end{tabular}




\section{Materiales y procedimiento}

Para la evaluación del funcionamiento familiar se aplicó el cuestionario Family Assessment Device (FAD) elaborado por Epstein y otros en 1983 -en base al modelo de funcionamiento familiar McMaster (McMaster Model of Family Functioning - MMFF)el cual fue validado para la población en estudio. La validez del instrumento fue obtenida a través del juicio de expertos, y la confiabilidad mediante coeficiente Alpha de Crombach que mide la consistencia interna arrojando un coeficiente total de 0.963 , valorado como muy alto, lo que indica que hay buena relación entre los İtems.

En cuanto a la variable rendimiento académico, se tomó como referencia el promedio de las notas obtenidas por los alumnos en el primer ciclo académico del 2008.

\section{RESULTADOS}

1. Tal como se observa en la Tabla2, en la presente investigación se encontró que el funcionamiento familiar, siguiendo al Modelo McMaster, es diferente en las familias de los estudiantes con alto y bajo rendimiento académico. La funcionalidad familiar, evaluada desde la percepción del alumno, mostró en la mayoría de las dimensiones un mejor funcionamiento para las familias del grupo con rendimiento académico alto, excepto en el funcionamiento de roles. Estos resultados podrían indicar la importante implicancia del funcionamiento familiar en la formación de hábitos de estudio y de apoyo emocional que requiere el estudiante universitario de los primeros años. Y que la familia, aún cuando los hijos asisten a las universidades, sigue jugando un rol importante.

2. Las familias de los estudiantes con alto y bajo rendimiento académico mostraron diferencias en la dimensión del compromiso afectivo según la prueba de independencia de criterios Chi cuadrada (p_value $0.000<0.05)$. De igual manera, el coeficiente $r$ de Spearman nos muestra un grado de intensidad de $r=$ 0.42 , p_value $=0.000$, que indica una buena correlación entre esta dimensión y el rendimiento académico. Evidentemente las familias de los alumnos con alto rendimiento académico valoran más las actividades de cada miembro, muestran más apoyo entre sí y se interesan en cada miembro en forma individual y empática, mientras que en las familias de los alumnos con bajo rendimiento académico según el MMFF, se muestran más narcisistas, egoístas e individualistas.

3. En cuanto a la dimensión de los Roles familiares se encontró que la relación según la prueba Chi cuadrado (p_value $0.062>0.05$ ) no es significativa. Si se toma en cuenta que lo roles familiares son patrones repetitivos por los que los miembros de la familia cumplen sus respectivas funciones, que incluyen: la toma de decisiones, un correcto liderazgo, funciones de límites y pertenencia, el cumplimiento de las tareas del hogar; se resaltará la importancia de la presencia del hijo en el hogar.

4. En cuanto a control conductual, la prueba de independencia de criterios Chi cuadrada muestra que sí hay relación con el rendimiento académico ( $p \_$value $0.000<0.05$ ). De igual manera, el coeficiente $r$ de Spearman nos muestra un grado de intensidad de $r=$ 0.392 , p_value $=0.000$, que indica una buena correlación entre esta dimensión y el rendimiento académico. Los alumnos con un bajo rendimiento académico refieren que sus familias adolecen de pautas que regulen el comportamiento de sus miembros. Evidentemente, si no hay un buen control de la disciplina, los hijos crecen sin un panorama claro de lo que se espera de ellos, de cómo debe ser su comportamiento dentro y fuera del hogar.

5. Otra de las funciones familiares alteradas en las familias de los alumnos con bajo rendimiento académico es la Comunicación. Los resultados obtenidos con la prueba de independencia de criterios Chi cuadrada muestra que sí hay relación con el rendimiento académico ( $p \_v a l u e 0.000<0.05$ ). De igual manera, el coeficiente $r$ de Spearman nos muestra un grado de intensidad de $r=0.216, p \_$value $=0.003$ que indica una buena correlación entre esta dimensión y el rendimiento académico. Evidentemente, la comunicación en las familias de los estudiantes con bajo rendimiento académico es más encubierta, menos clara y directa.

6. En cuanto a la respuesta afectiva la prueba de independencia de criterios Chi cuadrada muestra que sí hay relación con el rendimiento académico ( $p \_v a l u e$ $0.000<0.05)$. De igual manera, el coeficiente $r$ de Spearman nos muestra un grado de intensidad de $r=$ 0.378 , p_value $=0.000$, que indica una buena correlación entre esta dimensión y el rendimiento 
Tabla 2

Análisis de la relación entre el funcionamiento familiar y el rendimiento académico de los estudiantes del primery segundo año de la Facultad de Ciencias Humanas y Educación de la Universidad Peruana Unión en el 2008-1

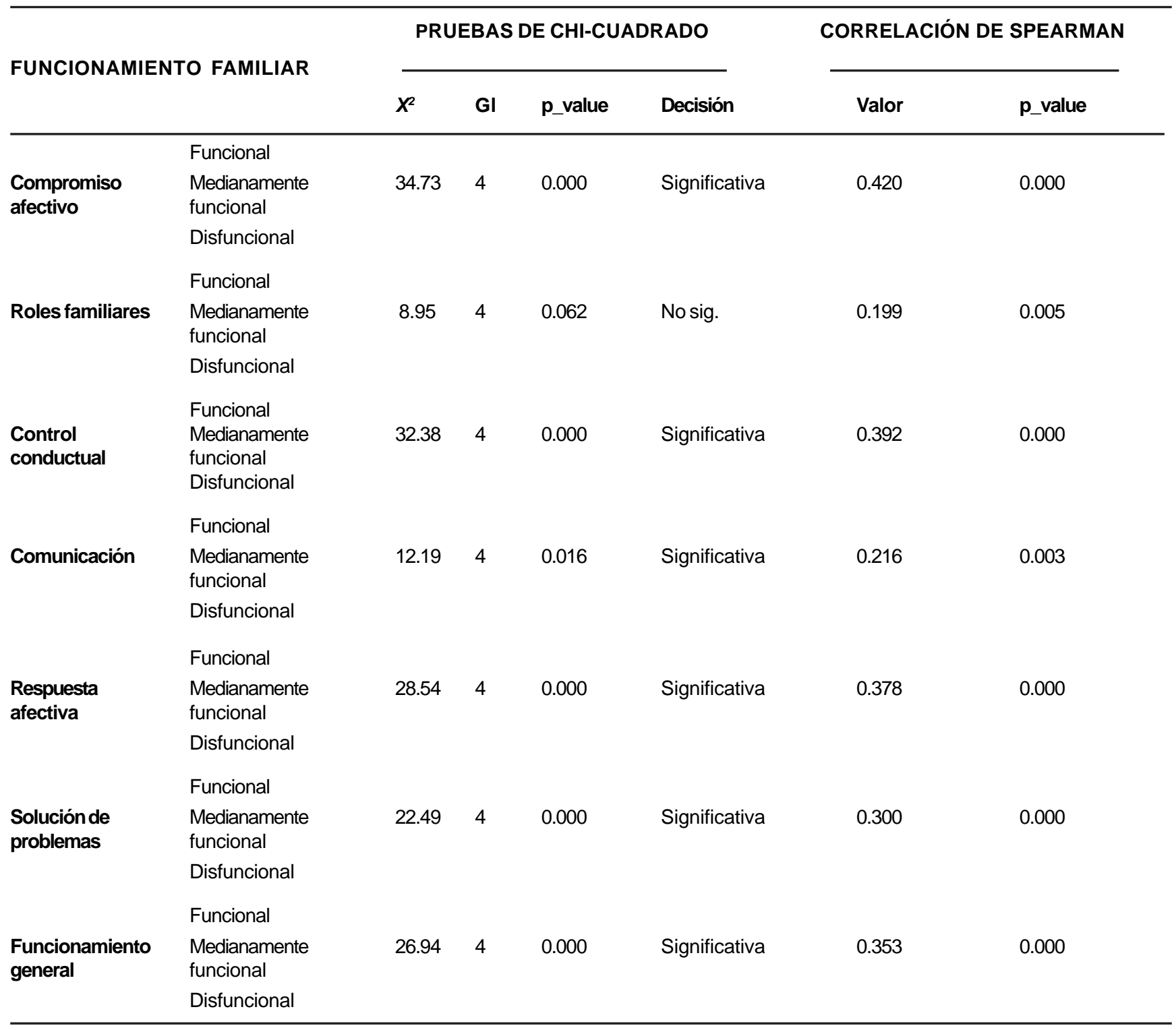

académico. Se deduce que la falta de habilidad en la expresión de las emociones tanto positivas como negativas, sumado a un estilo de comunicación deficiente, probablemente generen dificultades en las relaciones interpersonales y el aprendizaje del estudiante.

7. La disfuncionalidad encontrada en las familias de los alumnos con bajo rendimiento académico también es manifestada en la poca habilidad en el manejo y solución de los problemas cotidianos que les toca afrontar. Así la prueba de independencia de criterios Chi cuadrada muestra que sí hay relación entre la dimensión solución de problemas y el rendimiento académico ( $p \_$value $0.000<0.05$ ). De igual manera, el coeficiente $r$ de Spearman nos muestra un grado de intensidad de $r=0.30$, p_value $=0.000$, que indica una buena correlación entre esta dimensión y el rendimiento académico. Un aspecto que se debe tener presente es que un mayor nivel de funcionalidad no indica ausencia de problemas. No se trata de la cantidad de problemas que una familia tiene lo que la hace más efectiva que 
otra, sino el modo en que los problemas se encaran y solucionan.

8. La última dimensión, que resume y engloba los aspectos evaluados por las demás, es el funcionamiento general, donde la prueba Chi cuadrada muestra que sí hay relación con el rendimiento académico (p_value $0.000<0.05$ ). De igual manera, el coeficiente $r$ de Spearman nos muestra un grado de intensidad de $r=$ 0.353 que indica una buena correlación entre esta dimensión y el rendimiento académico ( $p \_$value $0.000<0.05$ ). Las diferencias se evidencian a través de una pobre contención afectiva, comunicación encubierta e indirecta, egoísmo y toma de decisiones infructuosas, aspectos que también sobresalieron en las demás áreas del funcionamiento familiar. Se coincide con lo planteado por el MMFF, de que la dinámica familiar es compleja y las dimensiones de su funcionamiento son complementarias y recíprocas.

\section{DISCUSIÓN}

Los resultados hallados indican que existe relación entre el funcionamiento familiar y el rendimiento académico de los alumnos del primer y segundo año de la facultad de Ciencias Humanas y Educación de la Universidad Peruana Unión, los cuales pueden ser comparados con el estudio de Andrade, Miranda y Freixas (2000), quienes estudiaron la predicción del rendimiento académico lingüístico y lógico matemático por medio de las variables modificables de las inteligencias múltiples y del hogar, llegando a la conclusión que efectivamente, existen relaciones significativas entre las variables intelectuales y del hogar con el rendimiento académico en alumnos de segundo año medio de la Comuna de Santiago en Chile.

Asimismo, Morris (1990) a través de un estudio intercultural encontró relación entre las dimensiones de la respuesta afectiva, roles, control conductual y funcionamiento general, lo cual se asemeja a los resultados obtenidos en nuestro trabajo ya que los puntajes obtenidos en cada dimensión para los grupos, divididos de acuerdo a su rendimiento académico, son similares. Se coincide con lo planteado por el MMFF, de que la dinámica familiar es compleja y las dimensiones de su funcionamiento son complementarias y recíprocas.
Valdés y otros (1997) analizaron las características del funcionamiento familiar que predicen conductas de riesgo en adolescentes y sus familias, identificando cuatro áreas que demostraron tener capacidad predictora de una menor ocurrencia en conductas de riesgo de los adolescentes, estas son: comunicación y conexión intrafamiliar, disponibilidad de los miembros de la familia, claridad y flexibilidad de roles y finalmente orden y jerarquía en la familia. En el presente estudio podemos apreciar resultados similares en relación a que un adecuado funcionamiento familiar en las dimensiones: comunicación, respuesta y compromiso afectivo, solución de problemas, control conductual y funcionamiento general podrían predecir y configurarse como factores protectores para un adecuado desempeño académico.

Los resultados obtenidos en esta investigación también se corroboran con lo hallado por Gonzáles y otros (2003) quienes analizaron la adaptabilidad y cohesión familiar, la implicación parental en conductas autorregulatorias, el autoconcepto del estudiante y el rendimiento académico, hallando que la percepción que los hijos tienen sobre el grado de implicación de los padres en comportamientos característicos autorregulatorios influye significativamente sobre las diferentes dimensiones que el estudiante tiene sobre sí mismo (vida privada, social y académica), y que este tipo de percepción se encuentra vinculada a las características de adaptabilidad y cohesión familiar.

Como se observa, es probable que una configuración de atributos de naturaleza negativa, que produce una atmósfera familiar más caótica y desequilibrada, tenga una fuerte repercusión conjunta sobre la conducta y el rendimiento académico del hijo universitario. Mientras que otra donde la afectividad, la disciplina, la comunicación y la solución de problemas es percibida por el joven como satisfactoria y eficaz, repercute de manera positiva en varios ámbitos de su desarrollo, como la vida académica.

Para finalizar se presentan las siguientes recomendaciones:

Réplica de la presente investigación en grupos de estudiantes de años superiores considerando otros factores que pueden incidir en el rendimiento académico del alumno tales como la estructura familiar, nivel socio económico, grado de instrucción de los padres, entre otros. 
Réplica de la investigación en contextos institucionales estatales y no religiosos y evaluar las diferencias en cuanto al funcionamiento familiar.

Observación del desempeño de los estudiantes en actividades distintas a las académicas y analizar la influencia del funcionamiento familiar en tales contextos.

Implementación de programas y actividades que fortalezcan los vínculos familiares de los estudiantes y que promuevan la integración exitosa de los alumnos de los primeros años, a la vida universitaria.

Motivación a los docentes universitarios para generar espacios de interacción con sus estudiantes, que faciliten la consejería y la expresión de inquietudes familiares y personales que predisponen el aprendizaje.

\section{REFERENCIAS}

Andrade, M., Miranda, C. y Freixas, I. (2000). Predicción del rendimiento académico lingüístico y lógico matemático por medio de las variables modificables de las inteligencias múltiples y del hogar. Contexto educativo, 3(17). Recuperado de http://www.unesco.cl/medios/biblioteca/ documentos/eaprendizajes_rendimiento _academico_2_medio_santiago.pdf?menu=/esp/ atematica/evalcontexinterc/docdig/

Baldwing, M. (1998). Modelo McMaster de evaluación familiar. Buenos Aires: Gedesa.

Cabrero, J. y Richart, M. (2008). Investigación no experimental, evaluativa y cualitativa. Apuntes de metodología de investigación. Recuperado de http:/ / perso.wanadoo.es/aniorte_nic/ apunt_metod_investigac4_6.htm

De la Cuesta, D., Pérez, E. y Louro, I. (1996). Funcionamiento familiar. Construcción y validación de un instrumento (Tesis de maestría). Universidad de La Habana, Cuba.

Epstein, N., Baldwing, L. \& Bishop, G. (1983). The McMAster Assessment Device. Journal of marital and family therapy, 9(2), 171-180. Recuperado de http://chipts.cch.ucla.edu/assessment/IB/ List_Scales/McMaster_Family- Assessment.htm

Garbanzo, V., G. (2007). Factores asociados al rendimiento académico en estudiantes universita- rios, una reflexión desde la calidad de la educación superior pública. Educación, 31(1), 43-63.

García, L. (1987). Rendimiento académico y abandono en la educación superior a distancia. Madrid: Universidad Nacional de Educación a Distancia.

González, J., Pienda, J., Núñez, C., Álvarez, L., Roces, C., González, S., González, P., Muñiz, R., Valle, A., Cabanach, R., Rodríguez, S. y Bernardo, A. (2003). Adaptabilidad y cohesión familiar, implicación parental en conductas autorregulatorias, autoconcepto del estudiante y rendimiento académico. Psicothema, 15(3), 471-477.

Hurtado, A. (2000). Familia y educación en el mundo de hoy. Revista de Educación, 10(5).

Morris, T. (1990). Culturally sensitive family assessment:an evaluation of the Family Assessment Device used with Hawaiian-American and JapanAmerican families. Family Process, 29, 105-116.

Ortiz, M. (1999). La salud familiar. Revista cubana de medicina general integral, 15(4). Recuperado de http://scielo.sld.cu/scielo.php?pid=S086421251999000400017\&script=sci_arttext\&tlng=es

Pizarro, R. (1985). Rasgos y actitudes del profesor efectivo(Tesis de maestría). Pontificia Universidad Católica de Chile, Chile.

Ridenour, T., Daley, J. \& Reich, W. (1999). Factor analyses of the Family Assessment Device. Family Process, 38, 497-510.

Sauceda, J. y Maldonado, J. (2003). La familia: su dinámica y tratamiento. Washington: Organización Panamericana de la Salud.

Universidad Nacional de Río Cuarto. (2008). Aprendiendo a ser universitario. Secretaría Académica, Ministerio de Educación, Ciencia y Tecnología de la Nación. Recuperado de www.eco.unrc.edu.ar/ingreso/inicio_vida_ universitaria.pdf

Valdés, M., Serrano, T., Rodríguez, J., Roizblatt, A., Florenzanoa, R. y Labra, J. (1997). Características del funcionamiento familiar que predicen conductas de riesgo en adolescentes y sus familias. FONDECYT. Recuperado de http://bases. bireme.br/cgi-bin/wxislind.exe/iah/online/ 